\title{
Monotone Comparative Statics of Characteristic Demand
}

\author{
Koji Shirai*†
}

January 27, 2010

\begin{abstract}
This study deals with comparative statics of the consumer's demand. According to Lancaster [1966, Journal of Political Economy], a utility function should be defined on the set of all characteristics that affect well-being of the consumer and these characteristics should be derived by consuming commodities. We show the sufficient condition for monotone comparative statics of the demand for characteristics and investigate the properties of the demand for commodities by considering the relationship between characteristics and commodities. We do not restrict the domain of a utility function and price systems, that is, the set of characteristics, to the Euclidean space. In particular, we allow discrete choice and nonlinear price systems. This theory enables us to predict the properties of the demand for the commodities that are not present in the market, such as new commodities. Our sufficient condition on a direct utility function can be characterized by the properties of the welfare variations for the change in the level of characteristics, which are more transparent and easier to verify. Further, the results of this study are derived by employing a new mathematical technique, which can be regarded as the generalization of the lattice theoretical comparative statics.
\end{abstract}

JEL classification: D11, C61.

Keywords: Monotone comparative statics; Characteristic demand; Consumer theory; Welfare variation; Value order.

*JSPS Research Fellow and Graduate School of Economics, Waseda University. This work was supported by Grant-in-Aid for JSPS Fellows

${ }^{\dagger}$ E-mail: shiraeconomics@suou.waseda.jp 


\section{Introduction}

In the standard consumer theory, the consumer has the preference on the set of commodities and he/she maximizes his/her preference subject to the linear budget constraint. Numerous studies along these lines have been conducted, and at least theoretically, various robust and sophisticated results have been revealed. However, as Lancaster (1966) points out, the standard theory has the only one answer to the following questions, "It is because of the tastes of the consumer."

"Why does the consumer choose to consume a particular good?"

"Why is some profile of goods prefered to the other ?"

"Why (or why not) is a good a close substitute for the other?".

That is because the conventional theory omits the intrinsic properties of goods and those of the consumer's preference.

Lancaster (1966) proposes an alternative approach to construct the consumer theory that includes these properties. Here, each profile of commodities is regarded as a profile of the characteristics that affect the well-being of the consumer, and preference is defined on the set of these characteristics. The characteristics are derived by consuming commodities, and hence, existing variations of commodities constitute the "physical constraint" for the consumer's choice. The price system of characteristics, which constitutes the "budget constraint" is derived from the relationship between the characteristics and commodities, and the price system of existing commodities. Therefore, in this theory, the consumer chooses the profile of characteristics that maximizes his/her preference subject to the budget constraint and the physical constraint. The solution set of the consumer's problem can be regarded as the "demand for characteristics," which can be translated into the demand for commodities by considering the relationship between commodities and characteristics. By employing this theory, we may answer the preceding 
questions along the lines of the following examples:

"A meal is consumed because it possesses nutritional characteristics that are essential for the consumer's life."

"Chocolates packed in an ornamental box are prefered to those packed in a plain cardboard box because the former provides the consumer with higher quality in appearance, while both possess the same level of taste and nutrition."

"A glass of Japanese whisky is a close substitute for a glass of Scotch whisky because these two goods share many characteristics."

However, it should be emphasized that this theory serves not only for providing the consumer theory with the heuristic basis but also for extending a more practical aspect of demand analyses. Specifically, as Lancaster states, it enables us to investigate or predict the consumer's demand for the goods or quality variations that have not yet emerged. Evidently, introducing new commodities is equal to altering the budget constraint and the physical constraint, and hence, the preference need not be modified. On the other hand, it is obvious that the standard theory cannot address such an analysis because the preference itself must be defined on the set of existing goods.

The idea of regarding a profile of commodities as a profile of characteristics has been successfully employed in the literature of the hedonic price studies initiated by Rosen (1974) and in its application in studies such as Palmquist (1984), which analyzes the housing demand by regarding a dwelling as a profile of relevant characterisitics. From the theoretical perspective, comparative statics analysis through this approach has been interested. Needless to say, comparative statics is an important topic in consumer theory since most of the empirical predictions of the consumer's behavior in response to the changes of some exogeneous parameters are derived from it. In addition, according to the statements in the previous paragraph, com- 
parative statics of characteristic demand enables us to predict the properties of the demand for new commodities, which is beyond the scope of standard consumer theory. The possible mathematical reasons behind the difficulty in constructing the theory on comparative statics for characteristic demand are presented as follows. First, if we defines the budget constraint on the set of characteristics, we must deal with the nonlinear constraint explicitly. Second, when considering the set of characteristics, we cannot expect that it is the Euclidean space, although most of researchers, including Lancaster and Rosen, implicitly regard it as the Euclidean space. This makes it difficult to justify the assumption of the smoothness and convexity of the preferences. With regard to the first aspect, in spite of Rosen's pessimistic prediction (Rosen (1974, p.59)), Edlefsen (1981) proposes a theory of comparative statics under nonlinear constraints. However, his theory depends on monotonicity and quasiconcavity of a utility function, twice continuous differentiablity of a utility function and constraints, and existence of an interior solution. Hence, the second aspect remains an open issue.

In this paper, we overcome the above difficulities by employing order theoretical comparative statics, which is often applied in recent economic theory, and investigate the condition for monotone comparative statics of characteristic demand, particularly, the monotonicity of income effects. The basis for constructing comparative statics is the "value order" method developed by Antoniadou (2007), Mirman and Ruble (2008), and Antoniadou et al. (2009). These studies discuss the monotone comparative statics of the consumer's demand along the lines of the standard consumer theory ${ }^{12}$. A brief description of the value order approach is as follows. First, some partial order that is suitable for comparative statics, the "value order," is defined on the consumption set (in this paper, the set of characteristics). Then, using this par-

\footnotetext{
${ }^{1}$ Nevertheless, Antoniadou et al. (2009) deal with the demand for lottery and provide the sufficient condition for the monotonicity of lottery demand in terms of the "quality" or the "characteristic" of the lottery like the first (second) order stochastic domination. In this sense, theoretically, our study can be regarded as an extension of their study.

${ }^{2}$ Quah (2007) presents another approach for order theoretical comparative statics of the consumer's demand. He dexterously shows the sufficient condition for monotone comparative statics by employing concavity and supermodularity of a utility function in the Euclidean space; which can be regarded as the generalization of Chipman (1977). However, in his approach, it is essential that the consumption set is a convex sublattice of $\mathbb{R}^{n}$.
} 
tial order, lattice theoretical comparative statics by Milgrom and Shannon (1994), and LiCalzi and Veinott (2005) is applied. Hence, the property called "quasisupermodularity" of a utility function with respect to the value order constitutes one of the sufficient conditions for monotone comparative statics. However, in this study, we do not employ lattice programming itself but generalized monotone comparative statics developed by Shirai $(2008)^{3}$. Although both the theories have fairly similar structures, the latter can be applied as long as some appropriate preorder is defined on the consumption set, while the former requires the consumption set to be a partially ordered set, more specifically, a lattice. In our general setting, it is difficult to construct an appropriate partial order. In particular, antisymmetry is quite difficult to be satisfied. Therefore, we employ new mathematical technique in this paper. As a result, we extend the value order method to the preordered sets and provide the sufficient condition for monotone comparative statics of the characteristic demand. Our requirement for the set of characteristics is that it should be written as the direct product of the set of each characteristic. Hence, we can allow the existence of characteristics that have only finite alternatives and those that cannot be expressed in terms of quantitative measure. Although we assume that the preference can be represented by a utility function, with regard to the minimum requirements for the domain of a utility function, differentiability, quasiconcavity, and similar regularity conditions in the standard consumer theory are not assumed. In addition, we do not impose concavity (convexity) assumptions on the price systems, although monotonicity and a weak form of continuity in the level of (at least) one characteristic is required.

While our sufficient condition on a utility function possesses high generality, it is inevitable that the more general the domain of a utility function is, the more opaque is the economic implication of the condition for a utility function. In this case, we must pay attention to the verifiablity of the sufficient condition. We can characterize the sufficient condition for a utility function in terms of the welfare variations for the change in a characteristic; this can

\footnotetext{
${ }^{3}$ See also Shirai (2009), which is a correction of Shirai (2008).
} 
be checked easily, as compared to the conditions on a direct utility function. In the literature of environmental economics, it is well known that the compensating variation, that is, the willingness to pay (WTP) for the change in an environmental quality can be estimated through the contingent valuation method, and that the monotonicity of WTP in income levels implies the necessary and sufficient condition for the normal demand, under the assumption of a unique solution and some regularity conditions (See McConnell (1990) and Whitehead (1995).). By applying our sufficient condition for a utility function we can extend this result to the context of characteristic demand without imposing regularity conditions such as differentiablity and strict quasiconcavity of a utility function, that are assumed in conventional WTP studies. Since the differentiability of a utility function in environmental qualities seems to be a rather technical assumption, in this sense, our results play a role of checking the robustness of the conventional WTP analysis. More precisely, we can interpret the above argument as a special case of the relationship between the sufficient condition based on the value order approach and the properties of welfare variations; this indicates the advantage of using the value order method.

The rest of this paper is organized as follows. In Section 2, our mathematical technique, that is, generalized monotone comparative statics based on Shirai (2008) is presented. Then, in Section 3.1, the formal definition of the consumer's problem in this study is defined. In Section 3.2, by applying the mathematics used in Section 2, the sufficient condition for monotone comparative statics is shown. The examples indicate that our formulation of the utility maximization problem, and hence our theory of comparative statics can encompass a wide range of consumer problems in economics, including the standard consumer theory. In Section 4, the sufficient condition for monotone comparative statics is characterized in terms of welfare variations. In Section 4.1, the general relationship between our sufficient condition and the properties of the welfare variations is investigated. In Section 4.2, by restricting the form of the price system, the relationship between our results and those of McConnell and Whitehead 
is explored.

\section{Mathematical Backgrounds}

In this section, we present our main mathematical technique to perform comparative statics analysis, as shown by Shirai (2008). It can be regarded as the generalization of the lattice theoretical comparative statics by Milgrom and Shannon (1994) and LiCalzi and Veinott (2005) in that it is equivalent to the conventional results when those can be applied. In the light of the mathematical structure of the comparative statics of consumer's problem, it seems natural to focus on comparative statics of the solution set of the maximization problem with respect to the changes of feasible sets ${ }^{4}$. Formally, the problem that we address can be written as follows. Let $X$ be the domain of the objective function $f: X \rightarrow \mathbb{R}$, that is, the whole set of alternatives, and let $S \subset X$ be a feasible set. Now, consider the maximization problem

$$
\max _{x \in S \subset X} f(x)
$$

Let $M(S)$ denote the solution set of the above problem when the feasible set is $S$. We intend to analyze the effects of the change of the feasible set from $S$ to $S^{\prime}$ on $M(\cdot)$. Specifically, in this study, monotone comparative statics forms the core of the analysis in this paper.

To facilitate the understanding of our technique, we first review the essence of the standard lattice theoretical comparative statics. The most fundamental requirement to employ the theory is that the domain of the objective function $X$ is a partially ordered set with special properties; which is refered to as a "lattice."

Definition 1: Let $\leq_{X}$ be a partial order on $X .\left(X, \leq_{X}\right)$ is a lattice if the infimum and the

\footnotetext{
${ }^{4}$ Milgrom and Shannon (1994) also consider the comparative statics with respect to the changes of objective functions.
} 
supremum of every two elements in $X$ exist. That is, $\left(X, \leq_{X}\right)$ is a lattice if both $x \wedge y=\sup \{z \mid$ $\left.z \leq_{X} x \& z \leq_{X} y\right\}$ and $x \vee y=\inf \left\{z \mid x \leq_{X} z \& y \leq_{X} z\right\}$ exist in $X$.

As can be presumed from what we are addressing, the partial order $\leq_{X}$ is the basis for the comparative criterion. That is, the comparative criterion that gauges the "changes," more specifically, the "monotonic changes," of feasible sets and the solution sets of maximization problems, is constructed from $\leq_{X}$.

Definition 2: Let $\left(X, \leq_{X}\right)$ be a lattice and $S, S^{\prime} \subset X$. We say that $S$ is lower than $S^{\prime}$ with respect to the strong set order, if $x \wedge y \in S$ and $x \vee y \in S^{\prime}$ for every $x \in S$ and $y \in S^{\prime}$. We denote this as $S \leq_{a} S^{\prime}$.

Note that, in general, $\leq_{a}$ is not reflexible. To derive reflexibility, a restriction should be imposed on $S$, which is stated as follows.

Definition 3: Let $\left(X, \leq_{X}\right)$ be a lattice. $S \subset X$ is said to be a sublattice of $X$ if $\left(S, \leq_{X}\right)$ is a lattice.

Milgrom and Shannon (1994) and LiCalzi and Veinott (2005) demonstrate that monotone comparative statics in terms of the strong set order is ensured if and only if the objective function $f: X \rightarrow \mathbb{R}$ satisfies the following condition.

Definition 4: Let $\left(X, \leq_{X}\right)$ be a lattice and $f: X \rightarrow \mathbb{R}$. We say that $f$ is quasisupermodular ${ }^{5}$

\footnotetext{
${ }^{5}$ LiCalzi and Veinott use the term lattice super extremum to refer to the same property. It should be noted that they also provide the necessary and sufficient condition for monotone comparative statics with respect to comparative criteria that are different from the strong set order.
} 
if

$$
f(x) \geq(>) f(x \wedge y) \Rightarrow f(x \vee y) \geq(>) f(y)
$$

Theorem 5 (Milgrom and Shannon, LiCalzi and Veinott): Let $X$ be a lattice and $S, S^{\prime} \subset X$. $M(S) \leq_{a} M\left(S^{\prime}\right)$ for every $S \leq_{a} S^{\prime}$ if and only if the objective function $f$ satisfies quasisupermodularity. In addition, if $S$ is a sublattice of $X$, then $M(S)$ is also a sublattice.

Proof See Milgrom and Shannon (1994), Theorem 4.

The construction of the preceding definitions and theorem clearly shows that to employ lattice theoretical comparative statics, it is essential that the domain of the objective function is a lattice, specifically, a partially ordered set. However, as stated in the previous section, under our general settings, it is difficult to define an appropriate partial order on the consumption set; Moreover, even if it was possible, the sufficient condition derived through Theorem 5 tends to be so restrictive that it has few economic implications. Hence, in this study, we employ generalized monotone comparative statics, which can be applied as long as an appropriate preorder is defined on the domain of the objective function. The following definitions imply that the concept of this theory is similar to the conventional lattice programming. The rest of this subsection is based on Shirai (2008). First, we define a preordered set with "lattice-like" properties, which is called a "preordered lattice structure."

Definition 6: Let $(X, \preccurlyeq X)$ be a preordered set. We say that $X$ is a preordered lattice structure if $T_{x, y} \neq \emptyset$ and $A_{x, y} \neq \emptyset$, where $T_{x, y}$ and $A_{x, y}$ is the set of the greatest lower bounds and that of the least upper bounds in $X$ respectively. 
Note that if $(X, \preccurlyeq X)$ is a partially ordered set, then a preordered lattice structure is a lattice. In this sense, it is an extension of the concept of a lattice. An important aspect to be noted is that both the greatest lower bounds and the least upper bounds with respect to the order on $X$ exist. Similar to the above case, we extend the notions of the strong set order and quasisupermodularity to the preordered lattice structure. If $\preccurlyeq x$ is a partial order, then each of the extended notions is equivalent with the corresponding notion in the standard lattice programming.

Definition 7: Let $(X, \preccurlyeq X)$ be a preordered lattice structure and $S, S^{\prime} \subset X$. We say that $S$ is lower than $S^{\prime}$ in terms of the $w$-strong set order if $T_{x, y} \cap S \neq \emptyset$ and $A_{x, y} \cap S^{\prime} \neq \emptyset$ for every $x \in S$ and $y \in S^{\prime}$. We denote this as $S \leq_{w a} S^{\prime}$. We say that $S$ is lower than $S^{\prime}$ in terms of the s-strong set order if $T_{x, y} \subset S$ and $A_{x, y} \subset S^{\prime}$ for every $x \in S$ and $y \in S^{\prime}$.

Definition 8: Let $(X, \preccurlyeq X)$ be a preordered lattice structure and $f: X \rightarrow \mathbb{R}$. We say that $f$ is w-quasisupermodular if

$$
\forall t \in T_{x, y} ; f(x) \geq(>) f(t) \Rightarrow \exists a \in A_{x, y} ; f(a) \geq(>) f(y)
$$

for every $x, y \in X$.

Note that both the w-strong set order and the s-strong set order do not satisfy reflexibility; this is similar to the case with the strong set order. To derive reflexibility, we define the counterparts of the concept of a sublattice.

Definition 9: Let $(X, \preccurlyeq X)$ be a preordered lattice structure. $S \subset X$ is said to be a w-sublattice of $X$ if $T_{x, y} \cap S \neq \emptyset$ and $A_{x, y} \cap S \neq \emptyset$ for every $x, y \in S . S \subset X$ is said to be an s-sublattice if $T_{x, y} \subset S$ and $A_{x, y} \subset S$ for every $x, y \in S$. 
Note that both $T_{x, y}$ and $A_{x, y}$ are taken with respect to $\preccurlyeq X . S \leq_{w a} S$ if and only if $S$ is a w-sublattice. The case with an s-sublattice is similar. Thus, we can extend Theorem 5 as follows.

Theorem 10: Let $(X, \preccurlyeq)$ be a preordered lattice structure and $f: X \rightarrow \mathbb{R}$. Let $M(S)$ be the solution set of the maximization problem

$$
\max _{x \in S \subset X} f(x)
$$

For every $S \leq_{s a} S^{\prime}, M(S) \leq_{w a} M\left(S^{\prime}\right)$ if and only if $f$ is w-quasisupermodular. In addition, if $S$ is an s-sublattice, then $M(S)$ is a w-sublattice.

Proof First, we demonstrate the sufficiency. Let $x \in M(S)$ and $y \in M\left(S^{\prime}\right)$. Since $S \leq_{s a} S^{\prime}$, $T_{x, y} \subset S$ and $A_{x, y} \subset S^{\prime}$. By definition, $f(x) \geq f(t)$ for all $t \in T_{x, y}$. Hence, by the wquasisupermodularity of $f$, there exists $a \in A_{x, y}$ such that $f(a) \geq f(y)$; this implies that $A_{x, y} \cap M\left(S^{\prime}\right) \neq \emptyset$. Similarly, $T_{x, y} \cap M(S) \neq \emptyset$. Otherwise, by the w-quasisupermodularity of $f$, there exists $a \in A_{x, y}$ such that $f(a)>f(y)$; this contradicts $y \in M\left(S^{\prime}\right)$.

Then, we show the necessity. Fix $x, y \in X$ and let $S=\{x\} \cup T_{x, y}$ and $S^{\prime}=\{y\} \cup A_{x, y}$ for $x, y \in X$. Obviously, $S \leq_{s a} S^{\prime}$. Suppose that $f(x) \geq f(t)$ for all $t \in T_{x, y}$. Then, $x \in M(S)$. Since $M(S) \leq_{w a} M\left(S^{\prime}\right)$, there exists $a \in A_{x, y}$ such that $a \in M\left(S^{\prime}\right)$; this implies that $f(a) \geq f(y)$. Suppose that $f(x)>f(t)$ for every $T_{x, y}$ and $y \in M\left(S^{\prime}\right)$. Then, there exists $t \in T_{x, y}$ such that $t \in M(S)$; this contradicts the assumption. Hence, there exists $a \in A_{x, y}$ such that $f(a)>f(y)$.

Suppose that $S$ is an s-sublattice. Let $x, y \in M(S)$. Since $S \leq_{s a} S$, by the result of the first paragraph of this proof, $M(S) \leq_{w a} M(S)$. [Q.E.D.] 


\section{Canonical Results}

In this section, we define the problem that we address in this paper and show the sufficent condition for monotone comparative statics of the consumer's demand. The formal definition of the consumer's problem is provided in the first subsection. In accordance with Lancaster (1966), a utility function of the consumer is defined on the set of characteristics or attributes that affect the well-being of the consumer. These characteristics are supposed to be derived by consuming the commodities, and hence, we consider the relationship between characteristics and commodities. In the second subsection, we proceed to comparative statics analysis by employing the mathematics that was used in the previous section. Our idea of monotone comparative statics is based on the "value-order" method developed by Antoniadou (2007) and Mirman and Ruble (2008). Further, we show that this approach can address a wide range of consumer's problems.

\subsection{Model}

In this subsection, we define the problem faced by the consumer. As stated at the start of this section, the preference of the consumer is defined on the set of attributes or characteristics derived through the consumption of commodities. Formally, let $X=X_{1} \times X_{2} \times \ldots \times X_{n}$ be the set of characteristics, where every $X_{k}$ is a set. For each profile of characteristics $x=$ $\left(x_{1}, x_{2}, \ldots, x_{n}\right) \in X$, the value of it is determined by a function $p: X \rightarrow \mathbb{R} \cup\{+\infty\}$, which is referred to as a price system in the rest of this paper. We assume that the preference of the consumer is represented by a utility function $U: X \rightarrow \mathbb{R}$. Then, given a price system $p(\cdot)$ and an income level $w>0$, the budget set of the consumer is $B(p, w)=\{x \in X \mid p(x) \leq w\}$, and the utility maximization problem is

$$
\max _{x \in B(p, w)} U(x) .
$$

Let $D(p, w)$ denote the solution set of this problem. To ensure the existence of the solution, we 
may assume the compactness of $B(p, w)$ and upper semi-continuity of $U$ with respect to some adequate topology. However, as far as comparative statics analysis, a topological property is not employed, and hence, the nonemptyness of $D(p, w)$ is directly assumed in the rest of this paper.

The commodities are taken into the above setting as follows. Let $\mathcal{M}$ be the set of variations of commodities, and let $Y \subset R_{+}^{\mathcal{M}}$ be a subset of the set of all nonnegative real-valued functions on $\mathcal{M}$. For instance, if $\mathcal{M}=\{1,2, \ldots, m\}$, then $Y$ can be written as a subset of $\mathbb{R}_{+}^{m}$. We regard $Y$ as a consumption set in terms of commodities. Note that we do not impose convexity on $Y$, and hence, the existence of indivisible commodities is allowed. Define the set-valued function $\mathcal{G}: Y \rightarrow 2^{X}$ such that $\mathcal{G}(y) \subset X$ represents the profiles of characteristics that are attainable from the profile of goods $y \in Y$ and let $\mathcal{G}^{-1}(x)=\{y \in Y \mid x \in \mathcal{G}(y)\}$. This is the generalization of the formulation in Lancaster (1966), where the relationship between the characteristics and the commodities is depicted by the linear transformation. In general, each profile of commodities $y$ can generate more than one profile of characteristics. This seems plausible if one assumes the "free disposal of characteristics," which is formally stated later. On the set $\mathcal{G}(Y) \subset X$, we define the price system of characteristics $p(x)$ as the value function of the minimization problem

$$
\min _{y \in \mathcal{G}^{-1}(x)} q(y)
$$

where $q: Y \rightarrow \mathbb{R}$ denotes the price system of commodities. If $Y \subset \mathbb{R}_{+}^{m}$ and the price of each commodity is separable and linear, $q(y)=q_{1} y_{1}+q_{2} y_{2}+\ldots+q_{m} y_{m}$. For $x \notin \mathcal{G}(Y)$, we suppose that $p(x)=+\infty$, which defines the physical constraint for the choice of the profile of characteristics. Thus, intuitively, $p(x)$ represents the minimal cost to enjoy the profile of characteristics $x$. Let $\Gamma(q, x)$ denote the solution set of the preceding minimization problem. If $x^{*} \in D(p, w)$, the set $\Gamma\left(q, x^{*}\right)$ is the demand for commodities corresponding to $x^{*}$. Thus, the demand for commodities corresponding to $D(p, w)$ is equal to $\cup_{x^{*} \in D(p, w)} \Gamma\left(q, x^{*}\right)=\Gamma(q, D(p, w))$. In the above argument, the mapping $\mathcal{G}$, and hence, $p(\cdot)$, could be different among consumers since 
they would reflect each consumer's characteristics.

Note that this model can encompass the standard consumer's problem by letting $X=Y$ and $\mathcal{G}$ be the identical mapping. In this sense, our setting can be seen as the generalization of the standard consumer theory. Moreover, our formulation of the utility maximization problem enables us to analyze the consumer's demand for the commodities that are not present in the market, such as new commodities. The appearance of new commodities causes the change of the feasible set of consumer. Let $\mathcal{M}^{\prime}$ be the altered set of variations of commodities and $Y^{\prime} \subset \mathbb{R}_{+}^{\mathcal{M}^{\prime}}$. Define $\mathcal{G}^{\prime}: Y^{\prime} \rightarrow 2^{X}$ as the set of profiles of characteristics that are attainable by $y \in Y^{\prime}$ and $\mathcal{G}^{\prime-1}: X \rightarrow 2^{Y^{\prime}}$ such that $\mathcal{G}^{\prime-1}(x)=\left\{y \in Y^{\prime} \mid x \in \mathcal{G}^{\prime}(y)\right\}$. Define $p^{\prime}: X \rightarrow \mathbb{R} \cup\{+\infty\}$ as the value function of the minimization problem

$$
\min _{y \in \mathcal{G}^{\prime-1}(x)} q^{\prime}(y)
$$

where $q^{\prime}(\cdot)$ is a price system of commodities defined on $Y^{\prime}$. Then, the demand for characteristics $D\left(p^{\prime}, w\right)$ is derived and, similar to the previous paragraph, it can be transformed into the demand for commodities, $\cup_{x^{*} \in D\left(p^{\prime}, w\right)} \Gamma\left(q^{\prime}, x^{*}\right)$. Intuitively, one can predict the demand for new commodities as long as their "properties" of them are known. This extension is impossible in the standard consumer theory. Indeed, in the conventional theory, a utility function or the preference of the consumer is defined on the set of existing commodities, say, $Y$, and hence, it is impossible to predict the reaction of the consumer to the new commodities.

Example 11: Consider the simple case in which there exist only two characteristics, the airconditioning capacity, $X_{1}$, and the composite characteristic that represents all other characteristics in the economy, $X_{2}$. Then, a utility function is $U: X \rightarrow \mathbb{R}$, where $X=X_{1} \times X_{2}$. Suppose that the characteristic of air-conditioning capacity is shared by only two commodities, for example, an electric fan (EF) and an air-conditioner (AC) and that the level of composite characteristic is equal to the consumption level of the numéraire composite good $y_{c} \in \mathbb{R}_{+}\left(=X_{2}\right)$. Then, 
the set of variations of commodities is $\mathcal{M}=\{E F, A C, C O M\}$, where $C O M$ denotes the composite good. For simplicity, we assume that the possible choice for the consumer with regard to the air-conditioning equipments is "to buy or not to buy" each commodity. That is, the set of possible profiles of commodities can be written as $Y=\{0,1\} \times\{0,1\} \times \mathbb{R}_{+}$. On the other hand, in terms of the characteristic of air-conditioning, the consumer can choose (1) no air-conditioning equipment $(\emptyset),(2)$ one unit of the electric fan $(E F),(3)$ one unit of the air-conditioner $(A C)$, and (4) one unit of the electric fan and the air-conditioner each $(E F \cup A C)$. Hence, the set of possible profiles of characteristics can be written as $\mathcal{G}(Y)=\{\emptyset, E F, A C, E F \cup A C\} \times \mathbb{R}_{+} \subset X$. Define the linear order ${ }^{6} \leq_{1}$ on $X_{1}$, which represents the level of the air-conditioning capacity. Assume that $\emptyset<_{1} E F<_{1} A C<_{1} E F \cup A C$ and free disposal of this characteristic is possible, that is, the level $E F$ can be achieved by $A C$ and $E F \cup A C$. The price system of characteristics $p: X \rightarrow \mathbb{R}$ can be constructed as follows. Let $q_{e}$ and $q_{a}$ be the unit price of EF and AC respectively. Suppose that $q_{e}<q_{a}$. For each $y_{c} \in \mathbb{R}_{+}$, the price system can be defined such that

$$
\begin{gathered}
p\left(\emptyset, y_{c}\right)=y_{c} \\
p\left(E F, y_{c}\right)=q_{e}+y_{c} \\
p\left(A C, y_{c}\right)=q_{a}+y_{c} \\
p\left(E F \cup A C, y_{c}\right)=q_{e}+q_{a}+y_{c} .
\end{gathered}
$$

For $x \in X \backslash \mathcal{G}(Y)$, define $p(x)=+\infty$.

Then, we consider introducing a new commodity, in particular, a new type of air-conditioning equipment (NAC). If we preserve the assumption of binary choice, the new set of profiles of commodities is $Y^{\prime}=\{0,1\} \times\{0,1\} \times\{0,1\} \times \mathbb{R}_{+}$. The new set of possible profiles of characteristics is $\mathcal{G}^{\prime}\left(Y^{\prime}\right)=\{\emptyset, E F, A C, N A C, E F \cup A C, E F \cup N A C, A C \cup N A C, E F \cup A C \cup N A C\} \times \mathbb{R}_{+} \subset X$.

\footnotetext{
${ }^{6} \mathrm{~A}$ binary relation is said to be a linear order or total order if it satisfies reflexibility, anti-symmetry, transitivity, and completeness.
} 
Let $q_{n}$ denote the unit price of NAC, and suppose that $q_{e}+q_{a}<q_{n}$. Assume that

$$
\begin{aligned}
& \emptyset<_{1} E F<_{1} A C<_{1}(E F \cup A C)<_{1} N A C<_{1} \\
&(E F \cup N A C)<_{1}(A C \cup N A C)<_{1}(E F \cup A C \cup N A C) .
\end{aligned}
$$

Then, the new price system $p^{\prime}$ can be defined such that, for each $y_{c} \in \mathbb{R}_{+}$,

$$
\begin{aligned}
p^{\prime}\left(\emptyset, y_{c}\right) & =y_{c} \\
p^{\prime}\left(E F, y_{c}\right) & =q_{e}+y_{c} \\
p^{\prime}\left(A C, y_{c}\right) & =q_{a}+y_{c} \\
p^{\prime}\left(E F \cup A C, y_{c}\right) & =q_{e}+q_{a}+y_{c} \\
p^{\prime}\left(N A C, y_{c}\right) & =q_{n}+y_{c} \\
p^{\prime}\left(E F \cup N A C, y_{c}\right) & =q_{e}+q_{n}+y_{c} \\
p^{\prime}\left(A C \cup N A C, y_{c}\right) & =q_{a}+q_{n}+y_{c} \\
p^{\prime}\left(E F \cup A C \cup N A C, y_{c}\right) & =q_{e}+q_{a}+q_{n}+y_{c} .
\end{aligned}
$$

Similar to the case with $p$, define $p^{\prime}(x)=+\infty$ for $x \in X \backslash \mathcal{G}\left(Y^{\prime}\right)$. Thus, even when a new commodity is introduced, the same utility function that was employed ealier can be used. In this case, the new price system $p^{\prime}(\cdot)$ is defined such that $p^{\prime}(x)=p(x)$, if $p(x)<+\infty$, although it need not be satisfied in general.

\subsection{Comparative Statics}

Now, we proceed to comparative statics of the demand for characteristics in our setting. 
In particular, we are interested in the sufficient condition under which the demand for characteristic $j$ is monotone "nondecreasing" in income levels. Monotone comparative statics of the demand for commodities is investigated by using the results on the characteristic demand. Since the set of characteristic $j, X_{j}$ is not necessarily a priori ordered, to make the notion of "nondecreasing" be well-defined, we must define some criterion $\leq_{j}$ that gauges the level of characteristic $j$. This is the generalization of $\leq_{1}$ in Example 11. In this study, we assume that the comparative criterion $\leq_{j}$ on $X_{j}$ is a linear order on $X_{j}$ and that it satisfies the following assumption.

Assumption 12: The comparative criterion $\leq_{j}$ is "value-increasing," that is, if $p\left(x_{-j}, x_{j}\right)<$ $+\infty, p\left(x_{-j}, x_{j}\right)<p\left(x_{-j}, x_{j}^{\prime}\right)$ for every $x_{-j} \in \times_{k \neq j} X_{k}$ and $x_{j}<_{j} x_{j}^{\prime}$. If $p\left(x_{-j}, x_{j}\right)=+\infty$, then $p\left(x_{-j}, x_{j}^{\prime}\right)=+\infty$ for $x_{j}<_{j} x_{j}^{\prime}$.

Note that Assumption 12 implicitly requires the possibility of "free disposal of characteristic $j . "$ Formally, free disposal of characterstic $j$ means that if $\left(x_{-j}, x_{j}^{\prime}\right)$ can be achieved by consuming a profile of goods $y$, then $\left(x_{-j}, x_{j}\right)$ is also attainable by $y$ for every $x_{j} \leq_{j} x_{j}^{\prime}$. If the assumption of free disposal fails to be satisfied, defining $p(x)=+\infty$ for every $x \notin \mathcal{G}(Y)$ may violate the preceding assumption. However, note that the assumption of free disposal does not necessarily imply the binding budget constraint. For instance, consider the case with discrete choice.

Since we do not assume the uniqueness of demand, the notion of "monotonicity" of the demand must be defined explicitly. Although there exist several notions of monotonicity ${ }^{7}$, we adopt the following.

Definition 13: Given a price system $p(\cdot)$, the demand for characteristic $j$ is pathwisely normal

\footnotetext{
${ }^{7}$ As an example, see Antoniadou (2007).
} 
with respect to $\leq_{j}$ if, for every $x \in D(p, w)$, there exists $x^{\prime} \in D\left(p, w^{\prime}\right)$ such that $x_{j} \leq_{j} x_{j}^{\prime}$ and, for every $x^{\prime} \in D\left(p, w^{\prime}\right)$, there exists $x \in D(p, w)$ such that $x_{j} \leq_{j} x_{j}^{\prime}$ for every $w<w^{\prime}$.

In the rest of this section, by applying the mathematical technique stated in the previous section, we show the sufficient condition for the demand for characteristic $j$ to be "normal" in the above sense. First, we impose the following assumption on the price systems of characteristics. Note that the condition is satisfied, for example, if there exists at least one characteristic $k(k \neq j)$ whose levels can be written as real numbers and a price system that is a continuous function in that characteristic.

Assumption 14: For every $\alpha \in \mathbb{R}$, if $p\left(x_{-j}, x_{j}\right)<\alpha$, then there exists some $x_{-j}(\alpha)$ such that $p\left(x_{-j}(\alpha), x_{j}\right)=\alpha$.

As already mentioned, our mathematical technique requires that some appropriate preorder is defined on the domain of the objective function. Under Assumptions 12 and 14, we can adopt the following.

Definition 15: Define $\preccurlyeq_{\left(p, \leq_{j}\right)}$ on $X$ such that $x \preccurlyeq_{\left(p, \leq_{j}\right)} x^{\prime}$ if $x_{j} \leq_{j} x_{j}^{\prime}$ and $p(x) \leq p\left(x^{\prime}\right)$. We refer to this as the $\left(p, \leq_{j}\right)$-value order. If $x_{j} \leq_{j} x_{j}^{\prime}$ and both $p(x)=+\infty$ and $p\left(x^{\prime}\right)=+\infty$, then we define that $x \preccurlyeq\left(p, \leq_{j}\right) x^{\prime}$.

This is the generalization of the notions of the "value orders" in Antoniadou (2007), Mirman and Ruble (2008), and Antoniadou et al. (2009) that are defined as partial orders. More precisely, our value order is the generalization of the "direct value order" in Antoniadou (2007). She defines it as a preorder on $\mathbb{R}_{+}^{n}$; however, in her analyses, she restricts the consumption set to $\mathbb{R}_{+}^{2}$ so that the direct value order satisfies antisymmetry. In addition to the direct value 
order, Mirman and Ruble (2008) introduce other value orders such as the "radial value order" and the "iterated value order"; in Antoniadou et al. (2009), the value orders that are beneficial for analyzing the lottery demands are defined. Note that it is common for every value order that it encompasses the comparative criterion and the value of the consumption bundles. It should be mentioned that, in our model, defining the appropriate partial order, particularly with regard to ensuring anti-symmetry, is quite difficult. Although, as long as $X=\mathbb{R}_{+}^{n}$, the iterated value order can be employed even when there exist more than two characteristics, the sufficient condition for normality based on that order is more restrictive in our setting; this is referred to later. The results in the next section also indicate the advantage of using the $\left(p, \leq_{j}\right)$-value order.

To confirm that the $\left(p, \leq_{j}\right)$-value order is suitable for our purpose, it must be shown that (1) the domain of a utility function, the set of characteristics, is a preordered lattice structure with respect to the $\left(p, \leq_{j}\right)$-value order, $(2)$ the budget set is s-strong set comparable, and (3) the w-strong set comparability of the demand set implies the pathwise normality of the demand for characteritic $j$ with respect to $\leq_{j}$. In our case, these properties are satisfied, as exlained below.

Proposition 16: Fix a price system $p(\cdot)$. Then, under Assumptions 12 and $14,\left(X, \preccurlyeq_{\left(p, \leq_{j}\right)}\right)$ is a preordered lattice structure.

Proof Let $x$ and $x^{\prime}$ be two incomparable points in $X$, with $x_{j}>_{j} x_{j}^{\prime}$ and $p(x)<p\left(x^{\prime}\right)$. Then, define $x_{j}^{t}$ such that $x_{j}^{t}=x_{j}^{\prime}$. By Assumption 12, $p\left(x_{-j}, x_{j}^{t}\right)<p\left(x_{-j}, x_{j}\right)$. Then, by Assumption 14, there exists $x_{-j}^{t}$ such that $p\left(x_{-j}^{t}, x_{j}^{t}\right)=p\left(x_{-j}, x_{j}\right)$. Obviously, this $x^{t}=$ $\left(x_{-j}^{t}, x_{j}^{t}\right)$ is an element of $T_{x . x^{\prime}}$. Similarly, define $x_{j}^{a}$ such that $x_{j}^{a}=x_{j}$. If $p\left(x^{\prime}\right)<+\infty$, since $p(x)=p\left(x_{-j}, x_{j}^{a}\right)<p\left(x_{-j}^{\prime}, x_{j}^{\prime}\right)=p\left(x^{\prime}\right)$, there exists $x_{-j}^{a}$ such that $p\left(x_{-j}^{a}, x_{j}^{a}\right)=p\left(x_{-j}^{\prime}, x_{j}^{\prime}\right)$ by Assumption 14. This $x^{a}=\left(x_{-j}^{a}, x_{j}^{a}\right)$ is an element of $A_{x, x^{\prime}}$. If $p\left(x^{\prime}\right)=+\infty$, by Assumption 12, 
$x^{a}=\left(x_{-j}^{\prime}, x_{j}\right)$ is also unattainable and it is an element of $A_{x, x^{\prime}}$. [Q.E.D.]

Proposition 17: Fix a price system $p(\cdot)$. Then, for every $w<w^{\prime}$, the budget sets are s-strong set comparable, that is, $B(p, w) \leq_{s a} B\left(p, w^{\prime}\right)$. In addition, for every $w$, the budget set is an s-sublattice of $X$.

Proof Let $x \in B(p, w)$ and $x^{\prime} \in B\left(p, w^{\prime}\right)$. Suppose that $x_{j}>_{j} x_{j}^{\prime}$ and $p(x)<p\left(x^{\prime}\right)$. However, for every element $x^{t} \in T_{x, x^{\prime}}$, we have $p\left(x^{t}\right)=p(x) \leq w$, which implies that $T_{x, x^{\prime}} \subset B(p, w)$. Similarly, $A_{x, x^{\prime}} \subset B\left(p, w^{\prime}\right)$.

Let $x, x^{\prime} \in B(p, w)$ with $x_{j}>_{j} x_{j}^{\prime}$ and $p(x)<p\left(x^{\prime}\right)$. However, for every $x^{t} \in T_{x, x^{\prime}}$, $p\left(x^{t}\right)=p(x) \leq w$, and for every $x^{a} \in A_{x, x^{\prime}}, p\left(x^{a}\right)=p\left(x^{\prime}\right) \leq w$. [Q.E.D.]

Proposition 18: Fix a price system $p(\cdot)$. Then, under Assumptions 12 and 14 , for every $w<w^{\prime}, D(p, w) \leq_{w a} D\left(p, w^{\prime}\right)$ implies that the demand for characteristic $j$ is pathwisely normal with respect to $\leq_{j}$.

Proof Let $x \in D(p, w)$ and $x^{\prime} \in D\left(p, w^{\prime}\right)$. Without loss of generality, we can suppose that $x_{j}>_{j} x_{j}^{\prime}$. By w-strong set comparability, there exists $x^{t} \in D(p, w) \cap T_{x, x^{\prime}}$. By definition of $T_{x, x^{\prime}}, x_{j}^{t}=x_{j}^{\prime}$. Similarly, there exists $x^{a} \in D\left(p, w^{\prime}\right) \cap A_{x, x^{\prime}}$ and $x_{j}^{a}=x_{j}$, which completes the proof. [Q.E.D.]

Then, by applying Theorem 10, we have the following.

Proposition 19: Fix a price system $p(\cdot)$. Then, under Assumptions 12 and 14, the demand for characteristic $j$ is pathwisely normal if a utility function $U$ satisfies w-quasisupermodularity with respect to the $\left(p, \leq_{j}\right)$-value order. In addition, $D(p, w)$ is a w-sublattice of the consumption 
set $X$ for every $w>0$.

Remark: In Mirman and Ruble (2008), as the generalization of the direct value order, the iterated value order is defined as follows. Let $X=\mathbb{R}_{+}^{n}$ and $p(x)=p_{1} x_{1}+p_{2} x_{2}+\ldots+p_{n} x_{n}$, where every $p_{k} \geq 0$. The iterated value order $\leq_{(p, i v)}$ is defined such that for every $x, x^{\prime} \in X$, $x \leq_{(p, i v)} x^{\prime}$ if $p_{1} x_{1} \leq p_{1} x_{1}^{\prime}, p_{1} x_{1}+p_{2} x_{2} \leq p_{1} x_{1}^{\prime}+p_{2} x_{2}^{\prime}, \ldots$, and $\sum_{k=1}^{n} p_{k} x_{k} \leq \sum_{k=1}^{n} p_{k} x_{k}^{\prime}$. It is shown that $\left(X, \leq_{(p, i v)}\right)$ is a lattice and if a utility function $U$ satisfies quasisupermodularity with respect to this order, the demand for $x_{1}$ is pathwisely normal. However, this sufficient condition depends on the numbering of coordinates and more restrictive than w-quasisupermodularity with respect to $\preccurlyeq\left(p, \leq_{1}\right)$. Indeed, it can be confirmed from the fact that for every $x, x^{\prime} \in X$, the infimum and the supremum with respect to $\leq_{(p, i v)}$ are elements of $T_{x, x^{\prime}}$ and $A_{x, x^{\prime}}$ with respect to $\preccurlyeq\left(p, \leq_{1}\right)$ respectively.

It should be noted that our sufficient condition does not require the regularity conditions that are usually assumed in the standard consumer theory. This is one of the advantages of the value order approach. The set of characteristics is not restricted to the Euclidean space; the price system is allowed to be nonlinear; a utility function is not assumed to be differentiable; the set valued demand is allowed; and the non-binding budget constraint is also allowed. Even in a simple model like Example 11, at least, the differentiable utility function is impossible to justify because of the discrete choice assumption. Although the condition "w-quasisupermodularity with respect to the $\left(p, \leq_{j}\right)$-value order" might seems to be opaque, in the next section, we show that the sufficient condition here can be characterized by the properties of the welfare variations that have transparent economic implications.

Example 11 (continued): In light of Proposition 19, given a price system $p(\cdot)$, the demand for the air-conditioning capacity is pathwisely normal if a utility function $U: X \rightarrow \mathbb{R}$ satisfies 
W-quasisupermodularity with respect to the $\left(p, \leq_{1}\right)$-value order. Recall that we assume that $\emptyset<_{1} E F<_{1} A C<_{1} E F \cup A C$. If the demand for the air-conditioning capacity is normal, we may observe that the demand for the air-conditioning commodities changes from $E F$ to $A C$, when the income level increases. Then, we can conclude that EF is an inferior good, which is correct. However, what is the reason bihind this phenomenon? According to the standard textbook of microeconomics, we may say that " $\mathrm{EF}$ is an inferior good because of the existence of a substitute with higher quality, $\mathrm{AC}^{\prime \prime}$. However, this interpretation seems inappropriate when we observes that a further increase in the income level leads to the shift from $\mathrm{AC}$ to $E F \cup A C$. In this situation, $\mathrm{EF}$ and $\mathrm{AC}$ can rather be regarded as complements. In addition, the term "substitute" and "complement" here indicate the intrinsic properties of goods that cannot be derived from the standard consumer theory. On the other hand, by using comparative statics of characteristic demand, we can make a well-defined interpretation such that "the demand shifts from EF to AC because the demand for the air-conditioning capacity is normal and AC provides the consumer with a higher level of that characteristic than EF," for instance.

From the above example, it is clear that comparative statics of characteristic demand also provides a rigorous interpretation for the income effects of the demand for commodities. Indeed, by extending the discussion in the previous example, we can show the sufficient condition under which the income effect of the demand for a particular commodity is nonnegative. An important point to be noted is that, by using the set-valued functions $\mathcal{G}$ and $\Gamma$, the demand for characteristics can be transformed into the demand for commodities. In the following statement, the normality of the demand for commodities can be defined in a similar manner that for the normality of the demand for characteristics.

Proposition 20: For every $x, x^{\prime} \in X, x_{j} \leq_{j} x_{j}^{\prime}$, and $\max \left\{p(x), p\left(x^{\prime}\right)\right\}<+\infty$, assume the

\footnotetext{
${ }^{8}$ Such statements can be found, for example, in Kreps (1990, p.49).
} 
following:

For every $y \in \Gamma(q, x)$, there exists $y^{\prime} \in \Gamma\left(q, x^{\prime}\right)$ such that $y(k) \leq y^{\prime}(k)$, and for every $y^{\prime} \in$ $\Gamma\left(q, x^{\prime}\right)$, there exists $y \in \Gamma(q, x)$ such that $y(k) \leq y^{\prime}(k)$.

Then, if the demand for characteristic $j$ is pathwisely normal, then the demand for commodity $k$ is also pathwisely normal.

Proof Let $w<w^{\prime}$ and $y \in \Gamma(q, D(p, w))$. Since the demand for characteristic $j$ is pathwisely normal, for every $x \in D(p, w)$, there exists $x^{\prime} \in D\left(p, w^{\prime}\right)$ such that $x_{j} \leq_{j} x_{j}^{\prime}$. By the assumption in the statement, there exists $y^{\prime} \in \Gamma\left(q, x^{\prime}\right)$ such that $y(k) \leq y^{\prime}(k)$. Obviously, $y^{\prime} \in \Gamma\left(q, D\left(p, w^{\prime}\right)\right)$. Similarly, it can be shown that, for every $y^{\prime} \in \Gamma\left(D\left(p, w^{\prime}\right)\right)$, there exists $y \in \Gamma(q, D(p, w))$ such that $y(k) \leq y^{\prime}\left(k^{\prime}\right)$. [Q.E.D.]

Intuitively, the condition in the preceding proposition implies that a higher number of commodity $k$ enables the consumer effeciently to acquire a higher level of characteristic $j$. In Example 11, $A C$ trivially satisfies this condition since $A C$ is essential to achieve the level of air-conditioning capacity that is higher than EF. According to our assumptions, if the new commodity, NAC, is introduced, we can predict that it will be a normal good, while AC will not be a normal good. The relationship between EF and AC (and NAC) in Example 11 also implies that the "substitutes" and "complements" in terms of intrinsic properties of commodities might be compatible. A commodity may be a close "substitute" of the other if these two goods share various characteristics. However, it is possible that these goods are "complements" with each other if the joint consumption leads to a high level or quality in terms of some characteristics ${ }^{9}$. Although we do not pursue this point in this paper, it might be an interesting topic for future

\footnotetext{
${ }^{9}$ In this sense, intrinsic complementarity seems to have the close relationship with monotonicity. Chambers and Echenique (2009) show the closeness between complementarity and monotonicity, from the mathematical viewpoint,.
} 
research.

Befere proceeding to the next section, we provide some examples to confirm that our expression of the utility maximization problem, and hence our comparative statics, can encompass a wide range of models.

Example 21: Let $X=\mathbb{R}_{+}^{n}$ and let the price system be linear. Then, $p\left(x_{1}, \ldots, x_{n}\right)=$ $p_{1} x_{1}+\ldots+p_{n} x_{n}$, which is the model of the standard consumer theory. This can be regarded as a special case of our setting in which it is supposed that each consumption good possesses peculiar and independent characteristics. In this example, each element in $X_{j}$ denotes the amount of consumption good $j$. If one intends to perform the quantitative comparative statics of the demand for good $j$, the comparative criterion $\leq_{j}$ is the Euclidean order. The $\left(p, \leq_{j}\right)$ value order $\preccurlyeq_{\left(p, \leq_{j}\right)}$ is defined such that $x \preccurlyeq_{\left(p, \leq_{j}\right)} x^{\prime}$ if $x_{j} \leq_{j} x_{j}^{\prime}$ and $p \cdot x \leq p \cdot x^{\prime}$. The same definition is used for the direct value order in Antoniadou (2007). By Proposition 19, if a utility function $U$ satisfies w-quasisupermodularity with respect to this order, the demand for good $j$ is pathwisely normal. Note that, if $n=2$, the direct value order is the partial order and standard lattice programming can be applied. Note that this argument can be applied even if there exist some indivisible commodities, as long as Assumptions 12 and 14 are satisfied.

Example 22: Consider the housing demand problem in Palmquist (1984). In this case, the consumer is supposed to purchase the numéraire composite good $x_{0} \in \mathbb{R}_{+}$and a dwelling. A dwelling is characterized as a profile of various characteristics $\left(x_{1}, \ldots, x_{n}\right) \in \times_{k=1}^{n} X_{k}$. Typically, the price of a dwelling is determined by a nonlinear function $p_{d}\left(x_{1}, \ldots, x_{n}\right)$, that is, a price system is written as $p(x)=x_{0}+p_{d}\left(x_{1}, \ldots, x_{n}\right)$. The consumer's utility is supposed to depend on the quantity of the composite good and the characteristics of the dwelling he/she purchases. This is similar to the setting in Example 11. However, in this case, it is supposed that a single commodity, that is, a dwelling, possesses more than one characteristic. The applicability of our 
sufficient condition, w-quasisupermdularity with respect to the value order, is obvious when one intends to confirm the normality of a particular characteristic of dwellings. In this example, the consumption set in terms of commodities is as follows. If there are $m$-types of dwellings, the set of variations $\mathcal{M}=\{0,1,2, \ldots, m\}$, where $0 \in \mathcal{M}$ denotes the composite good. Since we allow the consumer to choose only one dwelling, $Y$ can be written as

$$
Y=\left\{y \in \mathbb{R}_{+} \times\{0,1\}^{m} \mid y(k)=1 \Rightarrow y\left(k^{\prime}\right)=0, k, k^{\prime} \geq 1, \forall k \neq k^{\prime}\right\}
$$

Example 23: Consider the model of the consumer theory with uncertainty in Antoniadou et al. (2009). There exist two goods; one is deterministic and the other is subject to risk. The consumption set is defined as $X=\mathbb{R}_{+} \times \mathcal{F}_{y}$, where $\mathcal{F}_{y}$ is the set of distribution functions on $\mathbb{R}_{+}$. In general, the price of lottery is determined by a nonlinear function $p_{y}: \mathcal{F}_{y} \rightarrow \mathbb{R}_{+}$. Hence, a price system is written as $p(x, \tilde{y})=p_{x} x+p_{y}(\tilde{y})$. According to Antoniadou et al. (2009), we can consider the comparative criteria indicating the "quality" of lottery. In particular, consider the "first-order stochastic dominant" order $\leq_{F O S D}$ on $\mathcal{F}_{y}$. More precisely, for every $\tilde{y}, \tilde{y}^{\prime} \in \mathcal{F}_{y}$, $\tilde{y} \leq_{F O S D} \tilde{y}^{\prime}$ if $F_{y}(s) \geq F_{y^{\prime}}(s)$ for every $s \in \mathbb{R}_{+}$, where each $F_{y}$ and $F_{y^{\prime}}$ denote the distribution function corresponding to the lottery $\tilde{y}$ and $\tilde{y}^{\prime}$ respectively. However, $\leq_{F O S D}$ does not satisfy the completeness. Hence, we must restrict the consumption set to $X=\mathbb{R}_{+} \times \mathcal{F}_{y}^{C}$, where $\mathcal{F}_{y}^{C} \subset \mathcal{F}_{y}$ denotes a chain with respect to $\leq_{F O S D}$. Then, by Proposition 19, if a utility function satisfies w-quasisupermodularity with respect to the $\left(p, \leq_{F O S D}\right)$-value order, the demand for lottery is normal with respect to the first-order stochastic dominance order. This is nothing but the result in Antoniadou et al. (2009). Through the usage of the generalized monotone comparative statics (Theorem 10), it is possible to extend the model such that contains multiple lotteries without imposing any additional assumptions. 


\section{Welfare Variations and Normality}

In this section, we translate the sufficient condition in the previous section into more transparent and economic concepts. Its key concept is the welfare variations, namely, the compensating variation, and equivalent variation for the change in the level of a particular characteristic. In the first subsection, we explore the relationship between the sufficient condition for the normal demand and the properties of welfare variations in a general setting. Thus, we observe that the w-quasisupermodularity of a utility function with respect to the $\left(p, \leq_{j}\right)$-value order is characterized by the "single-crossing property" of the welfare variations. In the second subsection, we deal with the model in which the price system of characteristics is separable. It is shown that, in this case, one can confirm the normality of a particular characteristic only with limited information of the price system. More precisely, if the price of characteristic $j$ is independent of the level of other characteristics, one can derive the sufficient condition for the demand for characteristic $j$ to be normal without specifying the price system of that characteristic. In fact, our results can be regarded as the generalization of the results by McConnell (1990) and Whitehead (1995), that show the equivalency between normal demand and monotonicity of the willingness to pay, or the compensating variation, by employing the differentiability and strict quasiconcavity of a utility function. It is well known that the willingness to pay (the compensating variation) and the willingness to accept (the equivalent variation) can be estimated by the contingent valuation method, which is often employed in environmental economics. Hence, the results in this section imply that, in principle, our sufficient condition for the normality can be verified empirically.

\subsection{Welfare Variations and w-Quasisupermodularity}

Let us start by defining the welfare variations for the change in the level of a particular characteristic. Suppose that $x_{j}, x_{j}^{\prime} \in X_{j}$ and $x_{j}<_{j} x_{j}^{\prime}$. The compensating (equivalent) variation 
for the change from $x_{j}$ to $x_{j}^{\prime}$ is defined as follows. First, consider the maximization problem

$$
\max _{x_{-j} \in B_{-j}\left(p, w ; x_{j}\right)} U\left(x_{-j} ; x_{j}\right)
$$

where $B_{-j}\left(p, w ; x_{j}\right)=\left\{x_{-j} \in X_{-j} \mid p\left(x_{-j}, x_{j}\right) \leq w\right\}$. Let $V\left(p, w ; x_{j}\right)$ denote the value function of this problem. For simplicity, we refer to this as an indirect utility function. Note that the domain of $V\left(p, \cdot ; x_{j}\right)$ is the set of $w$ such that $B_{-j}\left(p, w ; x_{j}\right) \neq \emptyset$. Then, we define the welfare variations as follows.

Definition 24: Let $x_{j}<_{j} x_{j}^{\prime}$ and fix a price system $p(\cdot)$ and an income level $w>0$. The compensating variation for the change from $x_{j}$ to $x_{j}^{\prime}$ is defined as

$$
C\left(p, w, x_{j}, x_{j}^{\prime}\right)=\max \left\{c \mid V\left(p, w-c ; x_{j}^{\prime}\right)=V\left(p, w ; x_{j}\right)\right\} .
$$

Similarly, the equivalent variation for the change from $x_{j}$ to $x_{j}^{\prime}$ is defined as

$$
E\left(p, w, x_{j}, x_{j}^{\prime}\right)=\min \left\{e \mid V\left(p, w ; x_{j}^{\prime}\right)=V\left(p, w+e ; x_{j}\right)\right\} .
$$

Under Assumption 12, the domains of $C\left(p, \cdot, x_{j}, x_{j}^{\prime}\right)$ and $E\left(p, \cdot, x_{j}, x_{j}^{\prime}\right)$ are equal to the domain of $V\left(p, ; x_{j}^{\prime}\right)$.

Although the definitions of the welfare variations above appear to be similar to that in McConnell (1990) and Whitehead (1995), they slightly differ from the conventional definitions, as discussed in the next subsection. Note that the welfare variations in the above definition may be negative even if a utility function is increasing in $x_{j}$, since the higher level of characteristic $j$ straightens the budget set $B_{-j}$, that is,

$$
\left\{x_{-j} \in X_{-j} \mid p\left(x_{-j}, x_{j}\right) \leq w\right\} \subset\left\{x_{-j} \in X_{-j} \mid p\left(x_{-j}, x_{j}^{\prime}\right) \leq w\right\}
$$


for every $x_{j}<_{j} x_{j}^{\prime}$. Then, we intend to explore the two properties of the welfare variations with respect to income levels: nondecreasingness and the "single-crossing property". Both of these properties can be characterized in terms of the properties of an indirect utility function.

Proposition 25: Fix a price system $p(\cdot)$. Suppose that $w<w^{\prime}$ and $x_{j}<_{j} x_{j}^{\prime}$ satisfy $B_{-j}\left(p, w, x_{j}^{\prime}\right) \neq \emptyset$. Under Assumption 12, the compensating variation is nondecreasing in $w$, that is, $C\left(p, w, x_{j}, x_{j}^{\prime}\right) \leq C\left(p, w^{\prime}, x_{j}, x_{j}^{\prime}\right)$, if and only if $V$ satisfies

$$
V\left(p, w-c ; x_{j}^{\prime}\right) \geq(>) V\left(p, w ; x_{j}\right) \Rightarrow V\left(p, w^{\prime}-c ; x_{j}^{\prime}\right) \geq(>) V\left(p, w^{\prime} ; x_{j}\right)
$$

for every $c \in \mathbb{R}$. Similarly, the equivalent variation is nondecreasing in income levels if and only if the above condition is satisfied.

Proof First, we show the monotonicity of the compensating variation. Suppose that $V$ satisfies the condition in the statement. Then, $V\left(p, w-C\left(p, w, x_{j}, x_{j}^{\prime}\right) ; x_{j}^{\prime}\right)=V\left(p, w ; x_{j}\right)$, and hence, $V\left(p, w^{\prime}-C\left(p, w, x_{j}, x_{j}^{\prime}\right) ; x_{j}^{\prime}\right) \geq V\left(p, w^{\prime} ; x_{j}\right)$. This implies that $C\left(p, w, x_{j}, x_{j}^{\prime}\right) \leq C\left(p, w^{\prime}, x_{j}, x_{j}^{\prime}\right)$.

To show the converse, suppose that $V$ does not satisfy the condition in the statement. Then, there exist some $w<w^{\prime}$ and $x_{j}<_{j} x_{j}^{\prime}$ such that $V\left(p, w-c ; x_{j}^{\prime}\right) \geq(>) V\left(p, w ; x_{j}\right)$ and $V\left(p, w-c ; x_{j}^{\prime}\right)<(\leq) V\left(p, w ; x_{j}\right)$ for some $c \in \mathbb{R}$. This implies that $C\left(p, w, x_{j}, x_{j}^{\prime}\right) \geq(>) c$ and $C\left(p, w^{\prime}, x_{j}, x_{j}^{\prime}\right)<(\leq) c$, and hence, we have $C\left(p, w, x_{j}, x_{j}^{\prime}\right)>C\left(p, w^{\prime}, x_{j}, x_{j}^{\prime}\right)$.

The nondecreasingness of the equivalent variation can be shown as follows. Let $\bar{w}=$ $w-C\left(p, w, x_{j}, x_{j}^{\prime}\right)$ and $\bar{w}^{\prime}=w^{\prime}-C\left(p, w^{\prime}, x_{j}, x_{j}^{\prime}\right)$. Then, $w=\bar{w}+C\left(p, w, x_{j}, x_{j}^{\prime}\right)$ and $w^{\prime}=\bar{w}^{\prime}+$ $C\left(p, w^{\prime}, x_{j}, x_{j}^{\prime}\right)$. Note that $C\left(p, w, x_{j}, x_{j}^{\prime}\right)=E\left(p, \bar{w}, x_{j}, x_{j}^{\prime}\right)$ and $C\left(p, w^{\prime}, x_{j}, x_{j}^{\prime}\right)=E\left(p, \bar{w}^{\prime}, x_{j}, x_{j}^{\prime}\right)$. Since $C\left(p, w, x_{j}, x_{j}^{\prime}\right)$ is nondecreasing in income levels if and only if $V$ satisfies the condition in the statement, the monotonicity of $E$ also follows. [Q.E.D.]

Corollary 26: Suppose that an indirect utility function $V$ is increasing in $w$. Then, for given 
$x_{j}<_{j} x_{j}^{\prime}$, the absolute value of the equivalent variation is not smaller than the absolute value of the compensating variation if and only if both the compensating variation $C\left(p, w, x_{j}, x_{j}^{\prime}\right)$ and the equivalent variation $E\left(p, w, x_{j}, x_{j}^{\prime}\right)$ are nondecreasing in income levels.

Proof Under the assumption in the statement, the sign of the compensating variation and the welfare variation is the same with each other. Without loss of generality, we can suppose that $C\left(p, w, x_{j}, x_{j}^{\prime}\right)>0$. Assume that both the welfare variations are nondecreasing in $w$. For every $w>0$, define $\hat{w}=w+E\left(p, w, x_{j}, x_{j}^{\prime}\right)$. Similar to the proof of the preceding proposition, we have

$$
C\left(p, \hat{w}, x_{j}, x_{j}^{\prime}\right)=E\left(p, w, x_{j}, x_{j}^{\prime}\right)
$$

By assumption, $w<\hat{w}$, and hence,

$$
C\left(p, w, x_{j}, x_{j}^{\prime}\right) \leq C\left(p, \hat{w}, x_{j}, x_{j}^{\prime}\right)=E\left(p, w, x_{j}, x_{j}^{\prime}\right)
$$

To show the converse, suppose that $C\left(p, w, x_{j}, x_{j}^{\prime}\right)>E\left(p, w, x_{j}, x_{j}^{\prime}\right)$ for some $w>0$ and $x_{j}<_{j} x_{j}^{\prime}$. This implies that

$$
C\left(p, w, x_{j}, x_{j}^{\prime}\right)>C\left(p, \hat{w}, x_{j}, x_{j}^{\prime}\right)=E\left(p, w, x_{j}, x_{j}^{\prime}\right)
$$

This violates the nondecreasingness of $C\left(p, w, x_{j}, x_{j}^{\prime}\right)$. [Q.E.D.]

Then, we introduce the second property of welfare variations, namely, the single-crossing property, which is defined as follows.

Definition 27: Fix a price system $p(\cdot)$. The compensating variation $C\left(p, w, x_{j}, x_{j}^{\prime}\right)$ satisfies 
the single-crossing property in $\left(x_{j} ; w\right)$ if

$$
C\left(p, w, x_{j}, x_{j}^{\prime}\right) \geq(>) 0 \Rightarrow C\left(p, w^{\prime}, x_{j}, x_{j}^{\prime}\right) \geq(>) 0
$$

for every $w<w^{\prime}$ and $x_{j}<_{j} x_{j}^{\prime}$ satisfying $B_{-j}\left(p, w, x_{j}^{\prime}\right) \neq \emptyset$. The single-crossing property of the equivalent variation is similarly defined.

The term "single-crossing property" stems from the fact that if the welfare variation satisfies it, then it crosses 0 at most once from below. In fact, the single-crossing property of the welfare variations is equivalent with the single-crossing property of the indirect utility function $V\left(p, w ; x_{j}\right)$. Obviously, the single-crossing property is strictly weaker than the standard monotonicity, since every monotonic function trivially satisfies the single-crossing property. In addition, if the sign of a function is constant, then it satisfies the single-crossing property.

Proposition 28: Fix a price system $p(\cdot)$. The compensating variation satisfies the singlecrossing property in $\left(x_{j} ; w\right)$ if and only if $V$ satisfies the single-crossing property in $\left(x_{j} ; w\right)$, that is, for every $w<w^{\prime}$ and $x_{j}<_{j} x_{j}^{\prime}$ satisfying $B_{-j}\left(p, w, x_{j}^{\prime}\right) \neq \emptyset$,

$$
V\left(p, w ; x_{j}^{\prime}\right) \geq(>) V\left(p, w ; x_{j}\right) \Rightarrow V\left(p, w^{\prime} ; x_{j}^{\prime}\right) \geq(>) V\left(p, w^{\prime} ; x_{j}\right)
$$

Similarly, the equivalent variation satisfies the single-crossing property if and only if $V$ satisfies the single-crossing property in $\left(x_{j} ; w\right)$.

Proof First, we show the case with the compensating variation. Suppose that $V$ satisfies the single-crossing property in $\left(x_{j} ; w\right)$. Let $w<w^{\prime}$ and $C\left(p, w, x_{j}, x_{j}^{\prime}\right) \geq(>) 0$. This implies that $V\left(p, w ; x_{j}^{\prime}\right) \geq(>) V\left(p, w ; x_{j}\right)$. By the single-crossing property of $V$, we have $V\left(p, w^{\prime} ; x_{j}^{\prime}\right) \geq(>$ ) $V\left(p, w^{\prime} ; x_{j}\right)$. This implies that $C\left(p, w, x_{j}, x_{j}^{\prime}\right) \geq(>) 0$. 
To show the converse, suppose that $V$ does not satisfy the single-crossing property. Then, there exist some $w<w^{\prime}$ and $x_{j}<{ }_{j} x_{j}^{\prime}$ such that $V\left(p, w ; x_{j}^{\prime}\right) \geq(>) V\left(p, w ; x_{j}\right)$ and $V\left(p, w^{\prime} ; x_{j}^{\prime}\right)<$ $(\leq) V\left(p, w^{\prime} ; x_{j}\right)$. The former inequation $V\left(p, w ; x_{j}^{\prime}\right) \geq(>) V\left(p, w ; x_{j}\right)$ implies that $C\left(p, w, x_{j}, x_{j}^{\prime}\right) \geq$ $(>) 0$, while the latter inequation implies that $C\left(p, w, x_{j}, x_{j}^{\prime}\right)<(\leq) 0$.

The proof for the case with the equivalent variation is omitted because it is the same as that for the above argument, except that each of $C\left(p, w, x_{j}, x_{j}^{\prime}\right)$ and $C\left(p, w^{\prime}, x_{j}, x_{j}^{\prime}\right)$ are replaced with $E\left(p, w, x_{j}, x_{j}^{\prime}\right)$ and $E\left(p, w^{\prime}, x_{j}, x_{j}^{\prime}\right)$ respectively. [Q.E.D.]

With one additional assumption, we can characterize the w-quasisupermodularity of a utility function with respect to the $\left(p, \leq_{j}\right)$-value order by the single-crossing property of the welfare variations.

Assumption 29: For every $x_{j} \in X_{j}$, the maximization problem $\max _{B_{-j}\left(p, w, x_{j}\right)} U\left(x_{-j}, x_{j}\right)$ has at least one solution $x_{-j}^{*}$ such that $p\left(x_{-j}^{*}, x_{j}\right)=w$.

Theorem 30: Fix a price system $p(\cdot)$. Then, under Assumptions 12, 14, and 29, a utility function $U$ satisfies w-quasisupermodularity with respect to $\left(p, \leq_{j}\right)$-value order if and only if $V\left(p, w ; x_{j}\right)$ satisfies the single-crossing property in $\left(x_{j} ; w\right)$.

Proof First, we show the "if" part. Let $x, x^{\prime} \in X$. Suppose that $x_{j}>x_{j}^{\prime}$ and $p(x)<p\left(x^{\prime}\right)$. Since $\leq_{j}$ is a price increasing criterion, $x_{j}<_{j} x_{j}^{\prime}$. Define $x^{t^{*}}$ and $x^{a^{*}}$ such that

$$
\begin{gathered}
x_{-j}^{t^{*}} \in \underset{z_{-j} \in B_{-j}\left(p, p(x) ; x_{j}^{\prime}\right)}{\operatorname{argmax}} U\left(z_{-j} ; x_{j}^{\prime}\right), \\
x_{-j}^{a^{*}} \in \underset{z_{-j} \in B_{-j}\left(p, p\left(x^{\prime}\right) ; x_{j}\right)}{\operatorname{argmax}} U\left(z_{-j} ; x_{j}\right),
\end{gathered}
$$

with $x_{j}^{t^{*}}=x_{j}^{\prime}$ and $x_{j}^{a^{*}}=x_{j}$. By Assumption 29, we can assume that $p\left(x^{t^{*}}\right)=p(x)$ and 
$p\left(x^{a^{*}}\right)=p\left(x^{\prime}\right)$. It is obvious that $x^{t^{*}} \in T_{x, x^{\prime}}$ and $x^{a^{*}} \in A_{x, x^{\prime}}$. In the following, we prove that

$$
U(x) \geq(>) U\left(x^{t^{*}}\right) \Rightarrow U\left(x^{a^{*}}\right) \geq(>) U\left(x^{\prime}\right) .
$$

By the analogy of $x^{t^{*}}$ and $x^{a^{*}}$, we define $x^{*}$ and $x^{*}$ such that

$$
\begin{gathered}
x_{-j}^{*} \in \underset{z_{-j} \in B_{-j}\left(p, p(x) ; x_{j}\right)}{\operatorname{argmax}} U\left(z_{-j} ; x_{j}\right), \\
x_{-j}^{\prime *} \in \underset{z_{-j} \in B_{-j}\left(p, p\left(x^{\prime}\right) ; x_{j}^{\prime}\right)}{\operatorname{argmax}} U\left(z_{-j} ; x_{j}^{\prime}\right),
\end{gathered}
$$

with $x_{j}^{*}=x_{j}$ and $x_{j}^{*}=x_{j}^{\prime}$. Since $U\left(x^{t^{*}}\right)=V\left(p, p(x) ; x_{j}^{\prime}\right), U\left(x^{a^{*}}\right)=V\left(p, p\left(x^{\prime}\right) ; x_{j}\right), U\left(x^{*}\right)=$ $V\left(p, p(x) ; x_{j}\right)$, and $U\left(x^{\prime *}\right)=V\left(p, p\left(x^{\prime}\right) ; x_{j}^{\prime}\right)$, by the single-crossing property, we have

$$
\begin{aligned}
U\left(x^{*}\right) \geq(>) U\left(x^{t^{*}}\right) & \Longleftrightarrow V\left(p, p(x) ; x_{j}\right) \geq(>) V\left(p, p(x) ; x_{j}^{\prime}\right) \\
& \Rightarrow V\left(p, p\left(x^{\prime}\right) ; x_{j}\right) \geq(>) V\left(p, p\left(x^{\prime}\right) ; x_{j}^{\prime}\right) \\
& \Longleftrightarrow U\left(x^{a^{*}}\right) \geq(>) U\left(x^{* *}\right) .
\end{aligned}
$$

By definition, if $U(x) \geq(>) U(t)$ for all $t \in T_{x, x^{\prime}}$, then $U\left(x^{*}\right) \geq(>) U\left(x^{t^{*}}\right)$. The above inequations imply that $U\left(x^{a^{*}}\right) \geq(>) U\left(x^{\prime}\right)$ in such cases.

To show the converse, suppose that $V$ does not satisfy the single-crossing property. Without loss of generality, we can assume $V\left(p, w ; x_{j}^{\prime}\right) \geq(>) V\left(p, w ; x_{j}\right)$ and $V\left(p, w^{\prime} ; x_{j}^{\prime}\right)<(\leq$ ) $V\left(p, w^{\prime} ; x_{j}\right)$. Then, the inequations at the end of the previous paragraph imply that $U\left(x^{*}\right) \geq$ $(>) U\left(x^{t^{*}}\right)$ and $U\left(x^{a^{*}}\right)<(\leq) U\left(x^{*}\right)$. This implies that $U$ does not satisfy w-quasisupermodularity. [Q.E.D.]

Corollary 31: Fix a price system $p(\cdot)$. Then, under Assumptions 12, 14, and 29, the following 
statements are equivalent.

1. An indirect utility function $V$ satisfies the single-crossing property in $\left(x_{j} ; w\right)$.

2. The welfare variations satisfies the single-crossing property in $\left(x_{j} ; w\right)$.

3. A utility function satisfies w-quasisupermodularity with respect to the $\left(p, \leq_{j}\right)$-value order. Each of these statements implies that the demand for characteristic $j$ is pathwisely normal. In addition, if the demand for characteristic $j$ is unique, the converse also follows.

Corollary 32: Fix a price system $p(\cdot)$. Then, under Assumptions 12, 14, and 29, the following statements are equivalent.

1. An indirect utility function $V$ satisfies the condition

$$
V\left(p, w-c ; x_{j}^{\prime}\right) \geq(>) V\left(p, w ; x_{j}\right) \Rightarrow V\left(p, w^{\prime}-c ; x_{j}^{\prime}\right) \geq(>) V\left(p, w^{\prime} ; x_{j}\right)
$$

for every $w<w^{\prime}, x_{j}<_{j} x_{j}^{\prime}$ satisfying $B_{-j}\left(p, w, x_{j}^{\prime}\right) \neq \emptyset$.

2. For every $x_{j}<_{j} x_{j}^{\prime}$, the welfare variations are nondecreasing in $w$ as long as they are defined.

3. Suppose that $V$ is increasing in $w$. For every $x_{j}<_{j} x_{j}^{\prime}$, the absolute value of the equivalent variation is not smaller than the absolute value of the compensating variation (as long as they are defined).

Each of these statements implies the statements in the previous corollary, and hence, implies the normality of the demand for characteristic $j$.

The preceding corollaries imply that one can ensure the normality of the demand for a particular characteristic by investigating the properties of the welfare variations. It seems that the single-crossing property of an indirect utility function and the welfare variations are more 
transparent than the w-quasisupermodularity of a direct utility function with respect to the $\left(p, \leq_{j}\right)$-value order. However, it should be emphasized that employing the generalized monotone comparative statics, specifically, the $\left(p, \leq_{j}\right)$-value order, enables us to derive the sufficient conditions in terms of the welfare variations. The next subsection shows that our results can be regarded as an extension of the relationship between monotone comparative statics of the willingness to pay and monotone comparative statics of the demand; this technique is often applied in the literature of environmental economics, particularly in relation to the contingent valuation method. In this case the relationship between our definition of the welfare variations and the conventional welfare variations employed in McConnell (1990) and Whitehead (1995), plays an important role.

\subsection{In the Case of Separable Price Systems}

The sufficient conditions for the normality stated in the previous subsection depend on the specification of the price system of characteristics. On the other hand, as long as additive separability is imposed on the price systems, we can derive the sufficient condition for normality only with limited information of the price system. In the rest of this section, we assume that the price systems of characteristics can be written as $p\left(x_{-j}, x_{j}\right)=p_{-j}\left(x_{-j}\right)+p_{j}\left(x_{j}\right)$, which we refer to as the separability of the price of characteristic $j$. First, we slightly modify the definition of the welfare variations. For every $x_{j} \in X_{j}$, consider the maximization problem

$$
\max _{x_{-j} \in \tilde{B}_{-j}\left(p, w_{-j}\right)} U\left(x_{-j} ; x_{j}\right)
$$

where $\tilde{B}_{-j}\left(p, w_{-j}\right)=\left\{x_{-j} \mid p_{-j}\left(x_{-j}\right) \leq w_{-j}\right\}$. Let $\tilde{V}\left(p, w_{-j}, x_{j}\right)$ be the value function of the above problem. We refer to this as a conventional indirect utility function in order to distinguish it from an indirect utility function $V$ in the previous subsection. Needless to say, the domain of $\tilde{V}\left(p, \cdot ; x_{j}\right)$ is the set $\left\{w_{-j} \mid \tilde{B}_{-j}\left(p, w_{-j}\right) \neq \emptyset\right\}$. Then, in the followings, we define 
the welfare variations based on $\tilde{V}\left(p, w_{-j} ; x_{j}\right)$, which is the direct generalization of the definition of the welfare variations in McConnell (1990) and Whitehead (1995).

Definition 33: Let $x_{j}<_{j} x_{j}^{\prime}$. The conventional compensating variation for the change from $x_{j}$ to $x_{j}^{\prime}$ is defined as

$$
\tilde{C}\left(p, w_{-j}, x_{j}, x_{j}^{\prime}\right)=\max \left\{\tilde{c} \mid \tilde{V}\left(p, w_{-j}-\tilde{c} ; x_{j}^{\prime}\right)=\tilde{V}\left(p, w_{-j} ; x_{j}\right)\right\}
$$

Similarly, the conventional equivalent variation for the change from $x_{j}$ to $x_{j}^{\prime}$ is defined as

$$
\tilde{E}\left(p, w_{-j}, x_{j}, x_{j}^{\prime}\right)=\min \left\{\tilde{e} \mid \tilde{V}\left(p, w_{-j} ; x_{j}^{\prime}\right)=\tilde{V}\left(p, w_{-j}+\tilde{e} ; x_{j}\right)\right\}
$$

Note that, by definition, the conventional welfare variations are independent of the specification of the price of characteristic $j, p_{j}(\cdot)$. It should be also noted that, in contrast to the welfare variations in the previous subsection, the conventional welfare variations are always positive if a utility function is increasing in $x_{j}$.

Example 34: Consider the simplified version of the model of Whitehead (1995), which comprises three goods, namely, $x$ : the recreational use of a natural resource whose unit price is $p_{x}$, $q$ : the natural resource quality characteristic, and $z$ : the numéraire composite good. Consider the utility maximization problem

$$
\begin{gathered}
\max _{x, z} U(x, z ; q) \\
\text { s.t. } p_{x} x+z \leq w_{-q}
\end{gathered}
$$

and define its value function as $\tilde{V}\left(p, w_{-q} ; q\right)$. Then, the willingness to pay (WTP) for the change from $q_{0}$ to $q_{1}$ is defined as the conventional compensating variation, which in turn is 
defined as

$$
\tilde{C}\left(p_{x}, w_{-q}, q_{0}, q_{1}\right)=\max \left\{\tilde{c} \mid \tilde{V}\left(p, w_{-q}-\tilde{c} ; q_{1}\right)=\tilde{V}\left(p, w_{-q} ; q_{0}\right)\right\}
$$

for every $q_{0}<_{q} q_{1}$. In this example, the quality of the environment $q$ is nonmarketed good, and hence, one cannot observe the price system that explicitly includes $q$. McConnell (1990) and Whitehead (1995) prove that the monotonicity of $\tilde{C}$ in $\tilde{w}_{-j}$ for every $q_{0}<q_{1}$ is the necessary and sufficient condition for the normality of $q$ under every increasing $p_{q}>0$ with the assumptions of an increasing, strictly quasiconcave and twice continuously differentiable utility function.

As observed in the preceding example, if the sufficient condition for normality employing $\tilde{C}$ (or $\tilde{E}$ ) is constructed, one can perform comparative statics analysis only with limited information of price systems. In fact, provided the separability of the price of characteristic $j$ holds, we can extend the results of McConnell and Whitehead to our setting. To prove this, we clarify the relationship between comparative statics of the conventional welfare variations and a conventional indirect utility function. The proofs of the following two statements are omitted, since they are almost the same as those of Proposition 25 and Corollary 26 respectively.

Proposition 35: Fix a price system $p(\cdot)$ and suppose that the price of characteristic $j$ is separable and that $\tilde{B}_{-j}\left(p, w_{-j}\right) \neq \emptyset$. The conventional welfare variation is nondecreasing in $w_{-j}$, that is, $\tilde{C}\left(p, w_{-j}, x_{j}, x_{j}^{\prime}\right) \leq \tilde{C}\left(p, w_{-j}^{\prime}, x_{j}, x_{j}^{\prime}\right)$ if and only if $\tilde{V}$ satisfies

$$
\tilde{V}\left(p, w_{-j}-c ; x_{j}^{\prime}\right) \geq(>) \tilde{V}\left(p, w_{-j} ; x_{j}\right) \Rightarrow \tilde{V}\left(p, w_{-j}^{\prime}-c ; x_{j}^{\prime}\right) \geq(>) \tilde{V}\left(p, w_{-j}^{\prime} ; x_{j}\right)
$$

for every $x_{j}<_{j} x_{j}^{\prime}, w_{-j}<w_{-j}^{\prime}$, and $c \in \mathbb{R}$. Similarly, the conventional equivalent variation is nondecreasing in income levels if and only if the above condition is satisfied. 
Corollary 36: Suppose that a conventional indirect utility function $\tilde{V}$ is increasing in $w_{-j}$ and/or $x_{j}$. Then, for given $x_{j}<_{j} x_{j}^{\prime}$, the absolute value of the conventional equivalent variation is not smaller than the absolute value of the conventional compensating variation for every $x_{j}<_{j} x_{j}^{\prime}$ if and only if the conventional welfare variations are nondecreasing in $w_{-j}$.

Combined with Corollary 32, the following proposition ensures that the monotonicity of the conventional welfare variations under the price system $p(x)=p_{-j}\left(x_{-j}\right)+p_{j}\left(x_{j}\right)$ is the sufficient condition for the normality of characteristic $j$ for every price system $p^{\prime}(\cdot)$ such that $p^{\prime}(x)=p_{-j}\left(x_{-j}\right)+p_{j}^{\prime}\left(x_{j}\right)$ and satisfies Assumptions 12, 14, and 29.

Proposition 37: Fix a price system $p(\cdot)$ and suppose that the price of characteristic $j$ is separable. Then, under Assumptions 12 and 14, the conventional compensating (equivalent) variation $\tilde{C}\left(p, w_{-j}, x_{j}, x_{j}^{\prime}\right)\left(\tilde{E}\left(p, w_{-j}, x_{j}, x_{j}^{\prime}\right)\right)$ is nondecreasing in $w_{-j}$ if and only if $C\left(p^{\prime}, w, x_{j}, x_{j}^{\prime}\right)$ $\left(E\left(p^{\prime}, w, x_{j}, x_{j}^{\prime}\right)\right)$ is nondecreasing in $w$ for every price system $p^{\prime}(\cdot)$ such that $p_{-j}^{\prime}\left(x_{-j}\right)=$ $p_{-j}\left(x_{-j}\right)$.

Proof Let $w=w_{-j}+p_{j}\left(x_{j}\right)$. Then, $\tilde{V}\left(p, w_{-j} ; x_{j}\right)=V\left(p, w, x_{j}\right)$ and $\tilde{V}\left(p, w_{-j} ; x_{j}^{\prime}\right)=V(p, w+$ $\left.p_{j}\left(x_{j}^{\prime}\right)-p_{j}\left(x_{j}\right)\right)$. Hence, we have

$$
V\left(p, w+p_{j}\left(x_{j}^{\prime}\right)-p_{j}\left(x_{j}\right)-\tilde{C}\left(p, w_{-j}, x_{j}, x_{j}^{\prime}\right) ; x_{j}^{\prime}\right)=V\left(p, w ; x_{j}\right),
$$

implies that

$$
C\left(p, w, x_{j}, x_{j}^{\prime}\right)=\tilde{C}\left(p, w_{-j}, x_{j}, x_{j}^{\prime}\right)+p_{j}\left(x_{j}\right)-p_{j}\left(x_{j}^{\prime}\right)
$$

Hence, $C\left(p, w, x_{j}, x_{j}^{\prime}\right)$ is nondecreasing in $w$ if and only if $\tilde{C}\left(p, w_{-j}, x_{j}, x_{j}^{\prime}\right)$ in $w_{-j}$. In addition, $\tilde{C}\left(p, w_{-j}, x_{j}, x_{j}^{\prime}\right)$ is independent of the price of characteristic $j$, and hence, our claim follows. [Q.E.D.] 
Corollary 38: Fix a price system $p(\cdot)$ and suppose that the price of characteristic $j$ is separable. Then, under Assumptions 12, 14, and 29, the following statements are equivalent.

1. An indirect utility function $V$ satisfies

$$
V\left(p^{\prime}, w-c ; x_{j}^{\prime}\right) \geq(>) V\left(p^{\prime}, w ; x_{j}\right) \Rightarrow V\left(p^{\prime}, w^{\prime}-c ; x_{j}^{\prime}\right) \geq(>) V\left(p^{\prime}, w^{\prime} ; x_{j}\right)
$$

for every $p^{\prime}(\cdot)$ such that $p_{-j}^{\prime}(\cdot)=p_{-j}(\cdot), 0<w<w^{\prime}, x_{j}<x_{j}^{\prime}$ satisfying $B_{-j}\left(p, w, x_{j}^{\prime}\right) \neq \emptyset$, and $c \in \mathbb{R}$.

2. A conventional indirect utility function $\tilde{V}$ satisfies

$$
\tilde{V}\left(p, w_{-j}-c ; x_{j}^{\prime}\right) \geq(>) \tilde{V}\left(p, w_{-j} ; x_{j}\right) \Rightarrow \tilde{V}\left(p, w_{-j}^{\prime}-c ; x_{j}^{\prime}\right) \geq(>) \tilde{V}\left(p, w_{-j}^{\prime} ; x_{j}\right)
$$

for every $0<w_{-j}<w_{-j}^{\prime}, x_{j}<_{j} x_{j}^{\prime}$ satisfying $\tilde{B}_{-j}\left(p, w_{-j}\right) \neq \emptyset$ and $c \in \mathbb{R}$.

3. The welfare variations are nondecreasing in $w$ for every $x_{j}<_{j} x_{j}^{\prime}$ and $p^{\prime}(\cdot)$ such that $p_{-j}^{\prime}(\cdot)=p_{-j}(\cdot)$, as long as it is defined.

4. The conventional welfare variations are nondecreasing in $w_{-j}$ for every $x_{j}<_{j} x_{j}^{\prime}$ and $p^{\prime}(\cdot)$ such that $p_{-j}^{\prime}(\cdot)=p_{-j}(\cdot)$, as long as it is defined.

5. For every $x_{j}<_{j} x_{j}^{\prime}$, the absolute value of the conventional equivalent variation is not smaller than the absolute value of the conventional compensating variation under the condition that $\tilde{V}$ is increasing in $w_{-j}$ and/or $x_{j}$.

Each of these statements implies pathwise normality of the demand for characteristic $j$.

Although one may question the relationship between the single-crossing property of the conventional welfare variations and monotone comparative statics of characteristic demand, the single-crossing property does not necessarily imply normality. Indeed, even if $\tilde{C}$ satisfies 
the single-crossing property in $\left(x_{j} ; w_{-j}\right)$, the compensating variation $C$ does not necessarily satisfy the single-crossing property in $\left(x_{j} ; w\right)$. For instance, let $0<\tilde{C}\left(p, w_{-j}^{\prime}, x_{j}, x_{j}^{\prime}\right)<$ $\tilde{C}\left(p, w_{-j}, x_{j}, x_{j}^{\prime}\right)$ for some $w-p_{j}\left(x_{j}\right)=w_{-j}<w_{-j}^{\prime}=w^{\prime}-p_{j}\left(x_{j}\right)$, which does not violate the single-crossing property. However, in this case, it is possible that $C\left(p, w^{\prime}, x_{j}, x_{j}^{\prime}\right)<0<$ $C\left(p, w, x_{j}, x_{j}^{\prime}\right)$, since $\tilde{C}\left(p, w_{-j}, x_{j}, x_{j}^{\prime}\right)=C\left(p, w, x_{j}, x_{j}^{\prime}\right)+p_{j}\left(x_{j}\right)-p_{j}\left(x_{j}^{\prime}\right)$ and $p_{j}\left(x_{j}\right)-p_{j}\left(x_{j}^{\prime}\right)<0$.

Under a weak additional condition, the monotonicity of the welfare variations can be characterized in terms of the single-crossing property. That is, the following implies that the monotonicity of the conventional welfare variations is the necessary and sufficient condition for monotone income effects under the uniqueness of demand.

Proposition 39: Fix a price system $p(\cdot)$ and suppose that the price of characteristic $j$ is separable and that a utility function is increasing in $x_{j}$. Then, under Assumptions 12 and 14 , the conventional compensating (equivalent) variation $\tilde{C}\left(p, w_{-j}, x_{j}, x_{j}^{\prime}\right)\left(\tilde{E}\left(p, w_{-j}, x_{j}, x_{j}^{\prime}\right)\right)$ is nondecreasing in $w_{-j}$ if and only if $C\left(p^{\prime}, w, x_{j}, x_{j}^{\prime}\right)\left(E\left(p^{\prime}, w, x_{j}, x_{j}^{\prime}\right)\right)$ satisfies the single-crossing property in $\left(x_{j} ; w\right)$ for every price system $p^{\prime}(\cdot)=p_{-j}\left(x_{-j}\right)+p_{j}^{\prime}\left(x_{j}\right)$.

Proof We show the "only if" part. Suppose that $\tilde{C}\left(p, w_{-j}, x_{j}, x_{j}^{\prime}\right)>\tilde{C}\left(p, w_{-j}^{\prime}, x_{j}, x_{j}^{\prime}\right)$ for some $w_{-j}<w_{-j}^{\prime}$. Since a utility function is increasing in $x_{j}, \tilde{C}\left(p, w_{-j}, x_{j}, x_{j}^{\prime}\right)>0$. Define $p_{j}^{\prime}(\cdot)$ such that

$$
\begin{aligned}
& \tilde{C}\left(p, w_{-j}, x_{j}, x_{j}^{\prime}\right)>p_{j}^{\prime}\left(x_{j}^{\prime}\right)-p_{j}^{\prime}\left(x_{j}\right) \\
& \tilde{C}\left(p, w_{-j}^{\prime}, x_{j}, x_{j}^{\prime}\right)<p_{j}^{\prime}\left(x_{j}^{\prime}\right)-p_{j}^{\prime}\left(x_{j}\right) .
\end{aligned}
$$

Then, by letting $w=w_{-j}+p_{j}^{\prime}\left(x_{j}\right)$ and $w^{\prime}=w_{-j}+p_{j}^{\prime}\left(x_{j}\right), C\left(p^{\prime}, w, x_{j}, x_{j}^{\prime}\right)>0$ and $C\left(p^{\prime}, w^{\prime}, x_{j}, x_{j}^{\prime}\right)<$ 0 ; this violates the single-crossing property in $\left(x_{j} ; w\right)$.

The converse can be easily shown by letting $p^{\prime}=p$, and the case with the equivalent variation can be denoted in a similar fashion. [Q.E.D.] 
Corollary 40: Suppose that the price of characteristic $j$ is separable, and that a utility function is increasing in $x_{j}$. Fix a price system $p(\cdot)$. Then, under Assumptions 12, 14, and 29, the following statements are equivalent.

1. An indirect utility function $V$ satisfies the single-crossing property in $\left(x_{j} ; w\right)$.

2. An indirect utility function $V$ satisfies

$$
V\left(p, w-c ; x_{j}^{\prime}\right) \geq(>) V\left(p, w ; x_{j}\right) \Rightarrow V\left(p, w^{\prime}-c ; x_{j}^{\prime}\right) \geq(>) V\left(p, w^{\prime} ; x_{j}\right)
$$

for every $0<w<w^{\prime}, x_{j}<x_{j}^{\prime}$ satisfying $B\left(p, w, x_{j}^{\prime}\right) \neq \emptyset$ and $c \in \mathbb{R}$.

3. A conventional indirect utility function $\tilde{V}$ satisfies

$$
\tilde{V}\left(p, w_{-j}-c ; x_{j}^{\prime}\right) \geq(>) \tilde{V}\left(p, w_{-j} ; x_{j}\right) \Rightarrow \tilde{V}\left(p, w_{-j}^{\prime}-c ; x_{j}^{\prime}\right) \geq(>) \tilde{V}\left(p, w_{-j}^{\prime} ; x_{j}\right)
$$

for every $0<w_{-j}<w_{-j}^{\prime}, x_{j}<_{j} x_{j}^{\prime}$ satisfying $\tilde{B}_{-j}(p, w) \neq \emptyset$ and $c \in \mathbb{R}$.

4. The welfare variations satisfy the single-crossing property in $\left(x_{j} ; w\right)$ for every $p^{\prime}(\cdot)$ such that $p_{-j}^{\prime}(\cdot)=p_{-j}(\cdot)$.

5. The welfare variations are nondecreasing in $w$ for every $x_{j}<_{j} x_{j}^{\prime}$ and $p^{\prime}(\cdot)$ such that $p_{-j}^{\prime}(\cdot)=p_{-j}(\cdot)$.

6. The conventional welfare variations are nondecreasing in $w_{-j}$ for every $x_{j}<_{j} x_{j}^{\prime}$ and $p^{\prime}(\cdot)$ such that $p_{-j}^{\prime}(\cdot)=p_{-j}(\cdot)$, as long as it is defined.

7. For every $x_{j}<_{j} x_{j}^{\prime}$, the absolute value of the conventional equivalent variation is not smaller than the absolute value of the conventional compensating variation.

8. For every $x_{j}<_{j} x_{j}^{\prime}$, the absolute value of the equivalent variation is not smaller than the abosolute value of the compensating variation. 
9. A utility function $U$ satisfies w-quasisupermodularity with respect to the $\left(p^{\prime}, \leq_{j}\right)$-value order for every $p^{\prime}(\cdot)$ such that $p_{-j}^{\prime}(\cdot)=p_{-j}(\cdot)$.

Each of these statements implies pathwise normality of the demand for characteristic $j$. If the demand for characteristic $j$ is unique, the converse also follows.

As mentioned in Example 34, the conventional welfare variations $\tilde{C}\left(p, w_{-j}, x_{j}, x_{j}^{\prime}\right)$ and $\tilde{E}\left(p, w_{-j}, x_{j}, x_{j}^{\prime}\right)$ are clearly the willingness to pay and the willingness to accept respectively. In the literature of environmental economics, numerous studies have estimated these variables by using the contingent valuation method, and hence, it is possible, at least theoretically, to empirically verfy our sufficient condition in this subsection. Although the properties of the welfare variations in the previous subsection might be verified in a similar fashion, it should be noted that estimating the welfare variations $C$ and $E$ requires the specification of the price system of characteristics. In particular, the change of the set $B_{-j}(p, w ; \cdot)$ along with the change in the level of characteristic $j$ must be taken into account. Finally, we apply the results in this section to the examples.

Example 11 (continued): In this example, the price system can be written as $p\left(x_{1}, y_{c}\right)=$ $p_{1}\left(x_{1}\right)+y_{c}$, where $x_{1}$ denotes the level of air-conditioning capacity. Hence, the results in this subsection can be applied by our assumption on the price system of air-conditioning commodities. If the conventional welfare variations are nondecreasing in $w_{-1}$ for every $x_{1}<x_{1}^{\prime}$, the demand for air-conditioning capacity is pathwisely normal. Monotone comparative statics of the demand for commodities can be checked by applying Proposition 20. By our assumption, when $\mathrm{EF}$ and $\mathrm{AC}$ are the commodities that generate the air-conditining capacity, the latter satisfies the condition in Proposition 20, and hence, it is a normal good. If NAC is introduced in the economy, the demand for NAC is pathwisely normal, while the demand for AC is not 
normal in this case.

Example 21 (continued): Fix the unit prices $p_{k}(k \neq j)$. If the conventional welfare variations are nondecreasing in $w_{-j}$ for every $x_{j}<_{j} x_{j}^{\prime}$, then the demand for commodity $j$ is pathwisely normal for every $p_{j}>0$. If the demand for commodity $j$ is always unique, then the converse follows.

Example 22 (continued): Let $x_{j}$ be a qualitative characteristic of a dwelling. Since, in this example, the price system is not necessarily separable, we must employ the results of Section 4.1. If the welfare variations satisfy the single-crossing property in $\left(x_{j} ; w\right)$, then the demand for characteristic $j$ is pathwisely normal, and hence, the dwellings satisfying the condition of Proposition 20 are normal goods.

Example 23 (continued): Recall that, in this example, the price systems can be written as $p(x, \tilde{y})=p_{x} x+p_{y}(\tilde{y})$. Suppose that $p_{y}(\tilde{y})<p_{y}\left(\tilde{y}^{\prime}\right)$ if $\tilde{y}<_{F O S D} \tilde{y}^{\prime}$. By definition of the first-order stochastic dominance, a utility function is increasing with respect to $\leq_{F O S D}$. Thus, Corollary 40 can be applied. The demand for lottery is pathwisely normal for every increasing lottery price system $p_{y}^{\prime}(\cdot)$ if the conventional welfare variations for the improvements of the lottery in the sense of the first-order stochastic dominance is nondecreasing in $w_{-y}$. If the lottery demand is always unique, then the converse also follows. This result implies that the sufficient condition in Antoniadou et al. (2009), namely "the quasisupermodularity of a utility function with respect to the $\left(p, \leq_{F O S D}\right)$-value order," can be applied for every price system $p^{\prime}(\cdot)$ such that $p_{x}^{\prime}=p_{x}$ and satisfies Assumptions 12, 14, and 29. 


\section{References}

[1] Antoniadou, E. (2007): Comparative statics for the consumer problems. Economic Theory, 31(1), 189-203.

[2] Antoniadou, E., Mirman, L., Ruble, R. (2009): Lattices and lotteries. Mimeo.

[3] Chambers, C.P., Echenique, F. (2009): Supermodularity and preferences. Journal of Economic Theory, 144(3), 1004-1014.

[4] Chipman, J. (1977): An empirical implication of Auspitz-Lieben-Edgeworth-Pareto complementarity. Journal of Economic Theory, 14(1), 228-231.

[5] Edlefsen, L.E. (1981): The comparative statics of hednic price functions and other nonlinear constraints. Econometrica, 49(6), 1501-1520.

[6] Kreps, D.M. (1990): A course in microeconomic theory, Harvester Wheatsheaf, New York.

[7] Lancaster, K.J. (1966): A new approach to consumer theory. Journal of Political Economy, $74(2), 132-157$.

[8] Li Calzi, M.L., Veinott, A. (2005): Subextremal functions and lattice programming. Mimeo.

[9] McConnell, K.E. (1990): Models for referendum date: the structure of discrete choice models for contingent valuation. Journal of Environmental Economics and Management, $18(1), 19-34$.

[10] Milgrom, P., Shannon, C. (1994): Monotone comparative statics. Econometrica, 62(1), $157-180$.

[11] Mirman, L., Ruble, R. (2008): Lattice theory and the consumer's problem. Mathematics of Operations Research, 33, 301-314.

[12] Palmquist, R.B. (1984): Estimating the demand for the characteristics of housing. Review of Economics and Statics, 66(3), 394-404. 
[13] Quah, J.K-H. (2007): The comparative statics of constrained optimization problems. Econometrica, 75(2), 401-431.

[14] Rosen, S. (1974): Hedonic prices and implicit markets: Product defferentiation in pure competition. Journal of Political Economy, 82(1), 34-55.

[15] Shirai, K. (2008): A generalization of monotone comparative statics. Economics Bulletin, $3(39), 1-9$

[16] Shirai, K. (2009): A generalization of monotone comparative statics: A correction. Economics Bulletin, 29(1), 116-121.

[17] Whitehead, J.C. (1995): Willingness to pay for quality improvements: comparative statics and interpretation of contingent valuation results. Land Economics, 71(2), 207-215. 